\title{
Clinical characteristics in blood stream infections caused by Klebsiella pneumoniae, Klebsiella variicola, and Klebsiella quasipneumoniae: a comparative study, Japan, 2014-2017
}

Kazuo Imai ${ }^{1,2}$, Noriomi Ishibashi ${ }^{2,3}$, Masahiro Kodana ${ }^{4}$, Norihito Tarumoto ${ }^{1,2^{*}}$, Jun Sakai ${ }^{1,2}$, Toru Kawamura ${ }^{4}$, Shinichi Takeuchi ${ }^{4}$, Yoshitada Taji ${ }^{5}$, Yasuhiro Ebihara ${ }^{5}$, Kenji Ikebuchi ${ }^{4}$, Takashi Murakami ${ }^{2,6}$, Takuya Maeda ${ }^{2,6}$, Kotaro Mitsutake ${ }^{2,3}$ and Shigefumi Maesaki ${ }^{1,2}$

\begin{abstract}
Background: Klebsiella variicola and K. quasipneumoniae are new species distinguishable from K. pneumoniae but they are often misidentified as K. pneumoniae in clinical settings. Several reports have demonstrated the possibility that the virulence factors and clinical features differ among these three phylogroups. In this study, we aimed to clarify whether there were differences in clinical and bacterial features between the three phylogroups isolated from patients with bloodstream infections (BSIs) in Japan.

Methods: Isolates from all patients with BSIs caused by K. pneumoniae admitted to two hospitals between 2014 and $2017(n=119)$ were included in the study. Bacterial species were identified via sequence analysis, and their virulence factors and serotypes were analyzed via multiplex PCR results. Clinical data were retrieved from medical records.
\end{abstract}

Results: Of the 119 isolates, 21 (17.7\%) were identified as K. variicola and 11 (9.2\%) as K. quasipneumoniae; K1 serotype was found in $16(13.4 \%)$, and K2 serotype in $13(10.9 \%)$. Significant differences in the prevalence of rmpA, iutA, ybtS, ent $B$ and $k f u(p<0.001)$, and allS genes $(p<0.05)$ were found between the three phylogroups. However, there were no significant differences in clinical features, including the 30-day mortality rate, between the three organisms, although $K$. variicola was more frequently detected in patients over 80 years old compared with other Klebsiella species $(p<0.005)$, and K. quasipneumoniae more frequently occurred in patients with malignancy $(p<0.05)$.

Conclusions: Our findings demonstrated the differences in bacterial pathogenicity and clinical features among these three phylogroups. Further epidemiological studies into BSI caused by Klebsiella species are warranted.

Keywords: Klebsiella pneumoniae, Klebsiella variicola, Klebsiella quasipneumoniae, Blood stream infection, Japan

\footnotetext{
* Correspondence: tarumoto@saitama-med.ac.jp

'Department of Infectious Disease and Infection Control, Saitama Medical

University, 38 Morohongo, Moroyama-machi, Iruma-gun, Saitama 350-0495,

Japan

${ }^{2}$ Center for Clinical Infectious Diseases and Research, Saitama Medical

University, 38 Morohongo, Moroyama-machi, Iruma-gun, Saitama 350-0495,

Japan

Full list of author information is available at the end of the article
}

(c) The Author(s). 2019, corrected publication 2022 Open Access This article is distributed under the terms of the Creative Commons Attribution 4.0 International License (http://creativecommons.org/licenses/by/4.0/), which permits unrestricted use, distribution, and reproduction in any medium, provided you give appropriate credit to the original author(s) and the source, provide a link to the Creative Commons license, and indicate if changes were made. The Creative Commons Public Domain Dedication waiver (http://creativecommons.org/publicdomain/zero/1.0/) applies to the data made available in this article, unless otherwise stated. 


\section{Background}

Klebsiella pneumoniae is a frequent cause of infectious diseases in hospitals and community settings and is associated with a wide variety of clinical conditions including pneumonia, intra-abdominal infections, urinary tract infections, and bloodstream infections (BSIs). K. pneumoniae is reported to be the second most common cause of Gram-negative bacteremia and has a high mortality rate [1].

Recently, various microbiological factors and virulence genes of $K$. pneumoniae have been reported to be associated with the clinical features and a high mortality rate. Regarding the phenotypic features, it was demonstrated that a hypermucoviscous phenotype of $K$. pneumoniae could be a contributing factor in community-acquired primary liver abscesses $[2,3]$. The hypermucoviscous phenotype was previously common primarily in Asian countries, but it is now common worldwide [4]. Most isolates of the hypermucoviscous phenotype belong to the capsular K1 and $\mathrm{K} 2$ serotypes, and the predominant underlying mechanisms are due to the presence of regulator of mucoid phenotype A $(r m p A)$ and plasmid-borne regulator of extracellular polysaccharide synthesis (magA) [5-7]. Additionally, iron-scavenging systems (such as enterobactin, yersiniabactin, aerobactin $[8,9]$, and $k f u$ ion uptake system [10]), pili (type 3 fimbrial adhesin protein) [11], and allantoin metabolism [12] can also contribute to the virulence and the pathogenicity of K. pneumoniae.

The taxonomy of Klebsiella genus has been extensively studied, and $K$. variicola [13] and $K$. quasipneumoniae [14] were distinguished from K. pneumoniae as new species in 2004-previously, they were classified as members of $K$. pneumoniae phylogroups KpIII and KpII, respectively $[13,14]$. However, it is difficult to discriminate between these species using conventional laboratory methods because of their close phenotypic and biochemical features [15-17]. Recently, direct bacterial profiling via matrix-assisted laser desorption ionization-time of flight mass spectrometry (MALDI-TOF MS) has been established as a tool for the rapid identification of bacteria [18]. However, mass spectra derived from K. variicola and $K$. quasipneumoniae are very similar to the spectrum of $K$. pneumoniae. Therefore, these species have been erroneously identified as $K$. pneumoniae in clinical settings [17].

Maatallah and colleagues have reported that patients with $K$. variicola BSIs should be considered to be at higher risk of mortality than those with $K$. pneumoniae or K. quasipneumoniae BSIs [19]. However, few reports have evaluated the differences and the clinical impact between these three organisms; hence, further clinical investigation is needed.
In this study, we aimed to clarify differences in the clinical impact and bacterial characteristics of BSIs associated with $K$. pneumoniae, $K$. variicola, and K. quasipneumoniae in Japan from 2014 to 2017.

\section{Methods}

Patients and bacterial isolates

All patients with $K$. pneumoniae detected in blood samples who were admitted to Saitama Medical University Hospital and Saitama Medical University International Medical Center-a 1000-bed and a 700-bed tertiary care hospital and referral center, respectively-in Saitama, Japan, from 2014 to 2017 were included in this study. Only the first episode of bacteremia in each patient was included in this retrospective analysis, and 119 patients were finally enrolled. All isolates derived from blood cultures were identified by a MALDI Biotyper with MALDI Biotyper 3.1 software and MALDI Biotyper Reference Library version 4.0.0.1 (Bruker Daltonics, Bremen, Germany) according to the manufacturer's instructions in an autoflex speed mass spectrometer (Bruker Daltonics), which could not discriminate between $K$. variicola, K. quasipneumoniae, and K. pneumoniae [17], and stored at $-80^{\circ} \mathrm{C}$. Antimicrobial susceptibility testing was performed by Microscan Walk Away 96 Plus (Beckman Coulter, Brea, CA) and potential extended spectrum beta-lactamase (ESBL) producers were further tested and confirmed using a combined disk test according to the Clinical and Laboratory Standards Institute (CLSI) guidelines (document M100-S27).

\section{Clinical parameters}

A retrospective cohort study was conducted to evaluate the risk factors for mortality among the patients; 30-day mortality was the primary outcome measurement in this study. We collected patient information retrospectively from the electronic medical records of the hospitals. The data collected included the following: age, sex, patient risk factors (underlying disease, use of immunosuppressant and steroids, hemodialysis, and neutropenia; neutrophil count $<500$ cells $/ \mathrm{m}^{3}$ ), source of infection (which was reviewed retrospectively and described as "unknown" if not described in the medical records or determined by the attending physicians), polymicrobial bacteremia, use of catecholamines for septic shock, hospital-acquired infection versus community-acquired infection (infections were defined as hospital-acquired if the blood cultures were collected $>48 \mathrm{~h}$ after admittance to hospital or if the patient had been admitted to hospital within the previous 30 days), and appropriate or inappropriate antibiotic therapy within $24 \mathrm{~h}$ from the first BSI episode. Appropriate antibiotic therapy was defined as use of 
an antimicrobial agent to which isolates were susceptible based on in vitro susceptibility testing.

\section{Identification of K. variicola and K. quasipneumoniae from K. pneumoniae isolates}

The isolates, which were initially identified as $K$. pneumoniae using MALDI-TOF MS and stored until use, were identified by sequence analysis of subunit $\mathrm{C}$ of topoisomerase IV (parC) gene. Primer pairs for parC gene were used to identify $K$. variicola and $K$. quasipneumoniae according to previously reported protocols [20]. All isolates were cultured on MuellerHinton agar in advance. To prepare the template DNA for PCR, bacterial cells from a single colony were suspended in sterile distilled water and lysed at $98^{\circ} \mathrm{C}$ for $5 \mathrm{~min}$. The lysates were centrifuged at 13 , $000 \times g$ for $5 \mathrm{~min}$ and $1 \mu \mathrm{L}$ of supernatant was used for PCR reactions. Briefly, PCR amplicons were generated by TaKaRa Ex Taq (Takara Bio Inc., Gunma, Japan) and thermal cycling was carried out under the following conditions: $94^{\circ} \mathrm{C}$ for $5 \mathrm{~min}$, followed by 30 cycles at $98{ }^{\circ} \mathrm{C}$ for $10 \mathrm{~s}, 53^{\circ} \mathrm{C}$ for $30 \mathrm{~s}, 72^{\circ} \mathrm{C}$ for $30 \mathrm{~s}$, with a final extension at $72{ }^{\circ} \mathrm{C}$ for $5 \mathrm{~min}$. PCR products were analyzed using $1.2 \%(\mathrm{w} / \mathrm{v})$ agarose gel stained with ethidium bromide and purified using by ExoSAP-IT Express PCR Product Cleanup Reagent (Thermo Fisher Scientific, Massachusetts, USA). Sanger sequencing of these purified PCR amplicons was performed by Eurofines Genomics Co., Ltd. (Tokyo, Japan). Phylogenetic analysis was performed by MEGA 6 (https://www.megasoftware.net/). The phylogenetic tree was constructed by the neighbor-joining method based on the analysis of $319 \mathrm{bp}$ of the parC gene of 119 clinical isolates and reference strains. Klebsiella species were determined by identifying the cluster of the reference strains to which they belonged in the phylogenetic tree.

The $\operatorname{parC}$ gene sequence of reference strains were obtained from NCBI database (GenBank accession numbers: $K$. pneumoniae, CP009208, CP026586, NZ_AOCV00000000, NZ_CP015134 and NZ_CP030269; K. variicola, NZ_ CP017289 and NZ_CP030173; and K. quasipneumoniae, CP014696).

The reliability of the topology of the tree was checked by 500 bootstrap replications.

\section{Detection of virulence genes and serotyping}

To determine the capsular serotypes (K1 and K2) and to clarify the presence of genes associated with virulence factors (rmpA, entB, $y b t S$, iutA, $k f u, m r k D$, and allS), we used Multiplex-PCR, according to previously reported protocols [21]. ent $B, y b t S$, iut $A$, and mrkD encode enterobactin, yersiniabactin, aerobactin siderophore receptor, and the type 3 fimbrial adhesin protein, respectively. Furthermore, $k f u$ and alls are associated with the iron uptake system and allantoin metabolism, respectively. PCR amplicons were generated by KOD Multi-Epi (Toyobo, Osaka, Japan) and thermal cycling was carried out under the following conditions: $94^{\circ} \mathrm{C}$ for $5 \mathrm{~min}$, followed by 30 cycles at $98{ }^{\circ} \mathrm{C}$ for $10 \mathrm{~s}, 60^{\circ} \mathrm{C}$ for $30 \mathrm{~s}$, $68^{\circ} \mathrm{C}$ for $1 \mathrm{~min}$, with a final extension at $68^{\circ} \mathrm{C}$ for $5 \mathrm{~min}$. PCR products were analyzed using $2 \%(w / v)$ agarose gel stained with ethidium bromide.

\section{String test}

The hypermucoviscous phenotype of the isolates was determined using the string test, in which a standard bacteriological loop is used to stretch a mucoviscous string from each bacterial colony cultured on 5\% sheep blood agar. The formation of a viscous string $>5 \mathrm{~mm}$ in length was regarded as a positive test result [3].

\section{Statistical analysis}

Univariate analysis was performed to identify the differences in clinical characteristics between the three organisms. Univariate analysis was conducted using Fisher's exact test. Factors considered in univariate analysis were included in the multivariate analysis. Multivariable logistic regression analysis was used to assess the association between independent risk factors and mortality. All statistical analyses were calculated with $\mathrm{R}(\mathrm{v}$ 3.4.0; $\mathrm{R}$ Foundation for Statistical Computing, Vienna, Austria [http://www.R-project.org/]). A $p$-value of $<0.05$ was considered statistically significant.

\section{Results}

\section{Bacterial characteristics}

The characteristics of the isolates and the prevalence of virulence genes were characterized in Table 1 . Of the 119 isolates previously identified as $K$. pneumoniae by MALDI-TOF MS, 21 (17.7\%) were identified as $K$. variicola and 11 (9.2\%) as K. quasipneumoniae, based on sequence analysis of the parC gene (Additional file 1: Figure S1). Of the 119 isolates, 25 (21.0\%) were hypermucoviscous phenotype (K. pneumoniae $=22 \mathrm{~K}$. variicola $=2, K$. quasipneumoniae $=1$ ). The prevalence of rmpA and $\mathrm{K} 1$ and $\mathrm{K} 2$ serotypes was significantly higher in the hypermucoviscous phenotype isolates than in the non-hypermucoviscous phenotype isolates $(\mathrm{rmpA}: 76.0 \%$ vs $10.6 \%, p<0.001$; $\mathrm{K} 1: 32.0 \%$ vs $8.5 \%, p<0.005$; $\mathrm{K} 2$ : $28.0 \%$ vs $6.3 \%, p<0.005$ ) (Additional file 2: Table S2). Isolates of the hypermucoviscous phenotype belonging to $K$. variicola and $K$. quasipneumoniae were not $\mathrm{K} 1$ or K2 serotypes and did not have $r m p A$ gene (Additional file 2: Table S1).

Regarding the prevalence of the 7 virulence genes, rmpA and iutA were found only among $K$. pneumoniae isolates. Significant differences in the prevalence of the genes were found between $K$. pneumoniae, K. variicola, 
Table 1 Summary of bacterial characteristics for K. pneumoniae, K. variicola, and K. quasipneumoniae isolates

\begin{tabular}{|c|c|c|c|c|c|c|c|c|c|c|}
\hline & \multicolumn{2}{|c|}{ Total $=119$} & \multicolumn{2}{|c|}{ K. pneumoniae $=87$} & \multicolumn{2}{|c|}{ K. variicola $=21$} & \multicolumn{2}{|c|}{ K. quasipneumoniae $=11$} & \multirow{2}{*}{\multicolumn{2}{|c|}{$p$-value }} \\
\hline & $\bar{n}$ & $\%$ & $\bar{n}$ & $\%$ & $\bar{n}$ & $\%$ & $\bar{n}$ & $\%$ & & \\
\hline$\overline{\mathrm{K} 1}$ & 16 & (13.5) & 15 & $(17.2)$ & 0 & (0) & 1 & (9.1) & 0.09 & \\
\hline K2 & 13 & $(10.9)$ & 12 & $(13.8)$ & 1 & $(4.8)$ & 0 & (0) & 0.32 & \\
\hline$r m p A$ & 29 & $(24.4)$ & 29 & $(33.3)$ & 0 & (0) & 0 & (0) & $<0.001$ & * \\
\hline iutA & 32 & (26.9) & 32 & $(36.8)$ & 0 & $(0)$ & 0 & $(0)$ & $<0.001$ & * \\
\hline$y b t s$ & 45 & (37.8) & 41 & $(47.1)$ & 4 & $(19.0)$ & 0 & $(0)$ & $<0.001$ & * \\
\hline ent $B$ & 108 & (90.8) & 85 & $(97.7)$ & 19 & $(90.5)$ & 4 & $(36.4)$ & $<0.001$ & * \\
\hline$k f u$ & 45 & (37.8) & 26 & $(29.9)$ & 19 & $(90.5)$ & 0 & $(0)$ & $<0.001$ & * \\
\hline alls & 28 & (23.5) & 21 & $(24.1)$ & 1 & $(4.8)$ & 6 & $(54.5)$ & $<0.01$ & * \\
\hline$m r k D$ & 118 & $(99.2)$ & 86 & $(98.9)$ & 21 & $(100.0)$ & 11 & $(100.0)$ & 1.00 & \\
\hline Hypermucoviscous phenotype & 25 & $(21.0)$ & 22 & $(25.3)$ & 2 & $(9.5)$ & 1 & $(9.1)$ & 0.24 & \\
\hline ESBL-producing & 3 & $(2.5)$ & 2 & $(2.3)$ & 1 & (4.8) & 0 & (0) & 0.61 & \\
\hline
\end{tabular}

and $K$. quasipneumoniae isolates as follows: $r m p A$ (33.3\% vs $0 \%$ vs $0 \%, p<0.001)$, iutA $(36.8 \%$ vs $0 \%$ vs $0 \%, p<0.001), y b t S(47.1 \%$ vs $19.0 \%$ vs $0 \%, p<0.001)$, entB $(97.7 \%$ vs $90.5 \%$ vs $36.4 \%, p<0.001)$, $k f u(29.9 \%$ vs $90.5 \%$ vs $0 \%, p<0.001)$, and alls $(24.1 \%$ vs $4.8 \%$ vs $54.5 \%, p<0.05)$. Significant differences in the prevalence of $\operatorname{rmp} A$, iutA, entB, and $k f u$ virulence factors were found even when the isolates were divided into hospital-acquired and community-acquired infection groups (Additional file 2: Table S3).

Of the 119 isolates, $16(13.4 \%)$ belonged to the $\mathrm{K} 1$ serotype $(K$. pneumoniae $=15 ; K$. quasipneumoniae $=1$ ) and $13(10.9 \%)$ to the $K 2$ serotype $(K$. pneumoniae $=12$; $K$. variicola $=1)$. Only $2 K$. pneumoniae isolates $(2.3 \%)$ and $1 K$. variicola isolate $(4.7 \%)$ were identified as ESBL producers (Additional file 2: Table S1). K. pneumoniae isolates belonging to $\mathrm{K} 1$ serotype had all 7 virulence factors, while $K$. quasipneumoniae belonging to K1 serotype had only the allS and entB virulence factors (Additional file 2: Table S1).

\section{Clinical characteristics}

Of the 119 patients, 13 were excluded from the statistical analysis because of polymicrobial bacteremia. The characteristics of the patients are given in Table 2 and Additional file 2: Table S1. The most common underlying disease was malignancy (45 patients, $42.5 \%)$, which included both solid tumors and hematological malignant neoplasms. The most frequent source of bacteremia was abdominal infection (39 patients, 36.8\%), which included liver abscess (7 patients 6.6\%), urinary tract infections (31 patients, $29.2 \%$ ), pneumonia (6 patients, 5.7\%) and skin and soft tissue infections (4 patients, $3.8 \%$ ). None of the patients had complications of endophthalmitis or meningitis in this study. Of the 119 patients, 56 (52.8\%) had hospital-acquired infections. After the onset of bacteremia, 101 patients (95.3\%) were treated with appropriate antibiotic therapy within $24 \mathrm{~h}$. Eleven patients (10.3\%) died during the first 30 days.

\section{Differences in clinical characteristics of bacterial species}

The differences in the clinical characteristics among patients with $K$. pneumoniae, $K$. variicola, and $K$. quasipneumoniae infections are shown in Table 2. The median ages were 72,81 , and 77 years, respectively. K. variicola was the most frequent cause of BSI in patients over 80 years old $(p<0.005)$. BSIs with $K$. quasipneumoniae rather than $K$. pneumoniae and $K$, variicola $(p<0.05)$ frequently occurred in patients with malignancy. However, there were no significant differences in other characteristics (sex, source of infection, hospital or community acquired infection, or 30-day mortality) among patients with $K$. pneumoniae, $K$. variicola, or K. quasipneumoniae bacteremia. Regarding the phenotypes and prevalence of virulence genes, the hypermucoviscous phenotype was associated with intra-abdominal infection (odds ratio $[\mathrm{OR}]=2.8 ; 95 \%$ confidence interval $[\mathrm{CI}], 1.0-8.2 ; p<$ $0.05)$ and liver abscess $(\mathrm{OR}=26.7,95 \% \mathrm{CI}, 3.1-1335.6$; $p<0.001)$. However, there were no significant differences in the prevalence of virulence genes and serotypes of isolates based on clinical characteristics (Additional file 2: Table S4).

Differences in clinical characteristics between communityacquired and hospital-acquired infections

Of the 106 patients, 56 (52.8\%) were categorized as having hospital-acquired infections. The differences in the clinical characteristics among patients with community and hospital-acquired infections are shown in Table 3. Malignancy was more frequently associated with BSIs 
Table 2 Differences in clinical characteristics between patients with K. pneumoniae, K variicola, and K. quasipneumoniae bloodstream infections

\begin{tabular}{|c|c|c|c|c|c|c|c|c|c|c|}
\hline \multirow[t]{2}{*}{ Characteristics of patients } & \multicolumn{2}{|c|}{ Total $=106$} & \multicolumn{2}{|c|}{ K. pneumoniae $=78$} & \multicolumn{2}{|c|}{ K. variicola $=19$} & \multicolumn{2}{|c|}{ K. quasipneumoniae $=9$} & \multirow{2}{*}{\multicolumn{2}{|c|}{$p$-value }} \\
\hline & $\bar{n}$ & $\%$ & $\bar{n}$ & $\%$ & $\mathrm{n}$ & $\%$ & $\bar{n}$ & $\%$ & & \\
\hline Age, median, years & 74 & & 72 & & 81 & & 77 & & & \\
\hline Age $>80$ years & 29 & $(27.4)$ & 15 & $(19.2)$ & 11 & $(57.9)$ & 3 & (33.3) & $<0.005$ & * \\
\hline Male sex & 74 & $(69.8)$ & 53 & $(67.9)$ & 15 & $(78.9)$ & 6 & $(66.7)$ & 0.68 & \\
\hline Comorbidities & & & 0.0 & & & & & & & \\
\hline Diabetes mellitus & 29 & $(27.4)$ & 20 & $(25.6)$ & 7 & $(36.8)$ & 2 & $(22.2)$ & 0.61 & \\
\hline Malignancy & 45 & $(42.5)$ & 29 & $(37.2)$ & 9 & $(47.4)$ & 7 & (77.8) & $<0.05$ & * \\
\hline Liver cirrhosis & 11 & $(10.4)$ & 9 & $(11.5)$ & 2 & $(10.5)$ & 0 & (0) & 0.87 & \\
\hline Collagen disease & 8 & $(7.5)$ & 8 & $(10.3)$ & 0 & (0) & 0 & (0) & 0.36 & \\
\hline Chronic kidney disease & 4 & (3.8) & 4 & $(5.1)$ & 0 & (0) & 0 & (0) & 0.71 & \\
\hline Pulmonary disease & 4 & (3.8) & 4 & $(5.1)$ & 0 & (0) & 0 & (0) & 0.71 & \\
\hline Mental disorder & 7 & (6.6) & 5 & $(6.4)$ & 2 & $(10.5)$ & 0 & (0) & 0.80 & \\
\hline Immunosuppressive drug & 14 & $(13.2)$ & 12 & (15.4) & 2 & $(10.5)$ & 0 & (0) & 0.64 & \\
\hline Neutropenia & 7 & (6.6) & 5 & $(6.4)$ & 1 & $(5.3)$ & 1 & $(11.1)$ & 0.80 & \\
\hline \multicolumn{11}{|l|}{ Source of infection } \\
\hline Pneumonia & 6 & $(5.7)$ & 5 & (6.4) & 1 & (5.3) & 0 & (0) & 1.00 & \\
\hline Skin and soft tissue & 4 & (3.8) & 4 & $(5.1)$ & 0 & (0) & 0 & (0) & 0.70 & \\
\hline Abdominal & 39 & $(36.8)$ & 27 & (34.6) & 9 & $(47.4)$ & 3 & (33.3) & 0.40 & \\
\hline Urinary tract & 31 & $(29.2)$ & 26 & $(33.3)$ & 2 & $(10.5)$ & 3 & (33.3) & 0.14 & \\
\hline Liver abscess & 7 & (6.6) & 5 & $(6.4)$ & 2 & $(10.5)$ & 0 & (0) & 0.79 & \\
\hline \multicolumn{11}{|l|}{ Others } \\
\hline Hospital-acquired infection & 56 & $(52.8)$ & 41 & $(52.6)$ & 10 & $(52.6)$ & 5 & $(55.6)$ & 1.00 & \\
\hline Appropriate antibiotic therapy within $24 \mathrm{~h}$ & 101 & $(95.3)$ & 75 & $(96.2)$ & 17 & $(89.5)$ & 9 & (100) & 0.52 & \\
\hline
\end{tabular}

in patients with hospital-acquired infections compared with community-acquired infections $(53.6 \%$ vs $30.0 \%$; $p<0.05)$. All ESBL-producing $K$. pneumoniae were found in only patients with hospital-acquired infections (2.8\% vs $0 \%)$. There were no significant differences in the other characteristics (sex, source of infection, prevalence of virulence genes, antimicrobial susceptibility, or 30-day mortality) between patients with hospital-acquired infections and those with community-acquired infections (Table 3 and Additional file 2: Table S4).

\section{Risk factor analysis for 30-day mortality}

Of the 106 patients, 6 were excluded from the statistical analysis because we did not know the clinical courses of the patients during the 30 days following the onset of bacteremia. Risk factors found for 30-day mortality in univariate analysis were mental disorder $(\mathrm{OR}=7.7$; 95\% CI; 1.0-59.0, $p<0.05)$ and skin and soft-tissue infection $(\mathrm{OR}=$ 9.2; 95\% CI, 1.1-142.0; $p<0.05$ ) (Table 4). However, there were no significant differences in bacterial characteristics, including species differences, between survivors and non-survivors. In multivariate analysis, liver cirrhosis $(\mathrm{OR}=6.4 ; 95 \% \mathrm{CI}, 1.0-37.0 ; p<0.05)$, mental disorder $(\mathrm{OR}=15.0 ; 95 \% \mathrm{CI}, 2.33-97.1 ; p<0.005)$, and skin and soft-tissue infection $(\mathrm{OR}=13.2 ; 95 \% \mathrm{CI}$, $1.19-145.0 ; p<0.05)$ were found to be significant independent risk factors for 30-day mortality (Table 5).

\section{Discussion}

Previously, K. variicola and $K$. quasipneumoniae were often considered to be opportunistic pathogens with less virulence than $K$. pneumoniae in humans [14, 22]. However, recent studies have identified Klebsiella species on the basis of accurate molecular analysis and demonstrated that these pathogens are frequently detected at sites of infection in humans and in BSIs $[15,16]$. In our study, K. variicola and $K$. quasipneumoniae were isolated from BSI patients without underlying disease, and surprisingly, some fatal cases of bacteremia were caused by $K$. variicola and $K$. quasipneumoniae (Additional file 2: Table S1). Taking these findings into consideration, it is possible that the pathogenicity of both $K$. variicola and $K$. quasipneumoniae has been underestimated. 
Table 3 Differences in clinical characteristics between patients with hospital-acquired and community-acquired bloodstream infections

\begin{tabular}{|c|c|c|c|c|c|c|c|c|}
\hline \multirow[t]{2}{*}{ Characteristics of patients } & \multicolumn{2}{|c|}{ Total $=106$} & \multicolumn{2}{|c|}{ Hospital $=56$} & \multicolumn{2}{|c|}{ Community $=50$} & \multirow[t]{2}{*}{$p$-value } & \\
\hline & $n$ & $\%$ & $\mathrm{~N}$ & $\%$ & $\mathrm{n}$ & $\%$ & & \\
\hline Age, median, years & 74 & & 72 & & 75.5 & & & \\
\hline Male sex & 74 & $(69.8)$ & 43 & $(76.8)$ & 31 & $(62.0)$ & 0.14 & \\
\hline Age $>80$ years & 29 & $(27.4)$ & 12 & $(21.4)$ & 17 & $(34.0)$ & 0.19 & \\
\hline \multicolumn{9}{|l|}{ Comorbidities } \\
\hline Diabetes mellitus & 29 & $(27.4)$ & 13 & $(23.2)$ & 16 & $(32.0)$ & 0.38 & \\
\hline Malignancy & 45 & $(42.5)$ & 30 & $(53.6)$ & 15 & $(30.0)$ & $<0.05$ & * \\
\hline Liver cirrhosis & 11 & $(10.4)$ & 7 & $(12.5)$ & 4 & $(8.0)$ & 0.53 & \\
\hline Collagen disease & 8 & $(7.5)$ & 3 & $(5.4)$ & 5 & $(10.0)$ & 0.47 & \\
\hline Chronic kidney disease & 4 & $(3.8)$ & 1 & $(1.8)$ & 3 & (6.0) & 0.34 & \\
\hline Pulmonary disease & 4 & (3.8) & 1 & $(1.8)$ & 3 & $(6.0)$ & 0.34 & \\
\hline Mental disorder & 7 & $(6.6)$ & 4 & $(7.1)$ & 3 & $(6.0)$ & 1.00 & \\
\hline Immunosuppressive drug & 14 & $(13.2)$ & 6 & $(10.7)$ & 8 & $(16.0)$ & 0.57 & \\
\hline Neutropenia & 7 & $(6.6)$ & 5 & $(8.9)$ & 2 & $(4.0)$ & 0.44 & \\
\hline \multicolumn{8}{|l|}{ Source of infection } & \\
\hline Pneumonia & 6 & $(5.7)$ & 1 & $(1.8)$ & 5 & $(10.0)$ & 0.10 & \\
\hline Skin and soft-tissue & 4 & (3.8) & 2 & (3.6) & 2 & $(4.0)$ & 1.00 & \\
\hline Abdominal & 39 & $(36.8)$ & 18 & $(32.1)$ & 21 & $(42.0)$ & 0.32 & \\
\hline Urinary tract & 31 & $(29.2)$ & 15 & $(26.8)$ & 16 & $(32.0)$ & 0.67 & \\
\hline Liver abscess & 7 & $(6.6)$ & 3 & $(5.4)$ & 4 & (8.0) & 0.70 & \\
\hline \multicolumn{8}{|l|}{ Other } & \\
\hline Appropriate antibiotic therapy within $24 \mathrm{~h}$ & 101 & $(95.3)$ & 52 & $(92.9)$ & 49 & $(98.0)$ & 0.37 & \\
\hline \multicolumn{8}{|l|}{ Bacterial characteristics } & \\
\hline K. pneumoniae & 78 & $(73.6)$ & 41 & $(73.2)$ & 37 & $(74.0)$ & 1.00 & \\
\hline K. variicola & 19 & $(17.9)$ & 10 & $(17.9)$ & 9 & $(18.0)$ & 1.00 & \\
\hline K. quasipneumoniae & 9 & $(8.5)$ & 5 & $(8.9)$ & 4 & (8.0) & 1.00 & \\
\hline K1 serotype & 15 & $(14.2)$ & 7 & $(12.5)$ & 8 & $(16.0)$ & 0.78 & \\
\hline K2 serotype & 13 & $(12.3)$ & 6 & $(10.7)$ & 7 & $(14.0)$ & 0.77 & \\
\hline Hypermucoviscous phenotype & 23 & $(21.7)$ & 9 & $(16.1)$ & 14 & $(28.0)$ & 0.16 & \\
\hline ESBL-producing & 3 & $(2.8)$ & 3 & (3.4) & 0 & (0) & 0.38 & \\
\hline
\end{tabular}

CRBSI Catheter-related bloodstream infection

Our study showed differences in the phenotypic features and the prevalence of virulence genes between $K$. pneumoniae, $K$ variicola, and $K$. quasipneumoniae. Among the phenotypic features, the hypermucoviscous phenotype was found not only in isolates of K. pneumoniae but also in those of $K$. variicola and $K$. quasipneumoniae. The presence of rmpA has been reported to be associated with the hypermucoviscous phenotype. However, in this study, $\operatorname{rmp} A$ was confirmed only in isolates of $K$. pneumoniae and could not be confirmed in those of $K$. variicola or $K$. quasipneumoniae. In fact, the presence of strains negative for the $r m p A$ gene in hypermucoviscous phenotype isolates of $K$. variicola and $K$. quasipneumoniae has also been reported [23-25]. It was suggested that other mechanisms may contribute to the hypermucoviscous phenotype in these organisms.

In terms of virulence genes, significant differences in the prevalence of genes associated with iron-scavenging systems (entB, $y b t S$, iut $A$, and $k f u$ ) were recognized between $K$. pneumoniae, K. variicola, and K. quasipneumoniae isolates. The ability to acquire iron is essential for bacterial growth and replication, and the iron uptake system $(k f u)$ contributes to invasive disease and could play a part in the pathogenicity of BSIs caused by $K$. variicola [10]. In our results, the presence of $k f u$ was significantly higher in $K$. variicola isolates than in $K$. pneumoniae and $K$. quasipneumoniae isolates. It was previously reported that $K$. pneumoniae belonging to the 
Table 4 Univariate predictors of 30-day mortality among patients with bloodstream infections

\begin{tabular}{|c|c|c|c|c|c|c|c|}
\hline \multirow[t]{2}{*}{ Characteristics of patients } & \multicolumn{2}{|c|}{ Total $=100$} & \multicolumn{2}{|c|}{ Fatal $=11$} & \multicolumn{2}{|c|}{$\underline{\text { Survivors }=89}$} & \multirow[t]{2}{*}{$p$-value } \\
\hline & $n$ & $\%$ & $\mathrm{~N}$ & $\%$ & $n$ & $\%$ & \\
\hline Age, median, years & 75.0 & & 77.0 & & 75.0 & & \\
\hline Male sex & 70 & $(70.0)$ & 6 & $(54.5)$ & 64 & $(71.9)$ & 0.30 \\
\hline Age $>80$ years & 27 & $(27.0)$ & 4 & $(36.4)$ & 23 & $(25.8)$ & 0.48 \\
\hline \multicolumn{8}{|l|}{ Comorbidities } \\
\hline Diabetes mellitus & 29 & $(29.0)$ & 5 & $(45.5)$ & 24 & $(27.0)$ & 0.29 \\
\hline Malignancy & 41 & $(41.0)$ & 3 & $(27.3)$ & 38 & $(42.7)$ & 0.52 \\
\hline Liver cirrhosis & 10 & $(10.0)$ & 3 & $(27.3)$ & 7 & (7.9) & 0.08 \\
\hline Collagen disease & 8 & $(8.0)$ & 1 & $(9.1)$ & 7 & (7.9) & 1.00 \\
\hline Chronic kidney disease & 3 & (3.0) & 1 & $(9.1)$ & 2 & $(2.2)$ & 0.30 \\
\hline Pulmonary disease & 4 & $(4.0)$ & 1 & $(9.1)$ & 3 & (3.4) & 0.38 \\
\hline Mental disorder & 7 & $(7.0)$ & 3 & $(27.3)$ & 4 & $(4.5)$ & $<0.05$ \\
\hline Immunosuppressive drug & 12 & $(12.0)$ & 1 & $(9.1)$ & 11 & $(12.4)$ & 1.00 \\
\hline Neutropenia & 7 & $(7.0)$ & 0 & $(0)$ & 7 & (7.9) & 1.00 \\
\hline \multicolumn{8}{|l|}{ Source of infection } \\
\hline Pneumonia & 6 & $(6.0)$ & 0 & $(0)$ & 6 & $(6.7)$ & 1.00 \\
\hline Skin and soft-tissue & 4 & $(4.0)$ & 2 & $(18.2)$ & 2 & $(2.2)$ & $<0.05$ \\
\hline Abdominal & 37 & $(37.0)$ & 3 & $(27.3)$ & 34 & $(38.2)$ & 0.74 \\
\hline Urinary tract & 27 & $(27.0)$ & 2 & $(18.2)$ & 25 & $(28.1)$ & 0.72 \\
\hline \multicolumn{8}{|l|}{ Other } \\
\hline Hospital-acquired infection & 52 & $(52.0)$ & 6 & $(54.5)$ & 46 & $(51.7)$ & 1.00 \\
\hline Appropriate antibiotic therapy within $24 \mathrm{~h}$ & 96 & $(96.0)$ & 11 & $(100.0)$ & 85 & $(95.5)$ & 1.00 \\
\hline \multicolumn{8}{|l|}{ Bacterial characteristics } \\
\hline K. pneumoniae & 73 & $(73.0)$ & 8 & $(72.7)$ & 65 & $(73.0)$ & 1.00 \\
\hline K. variicola & 19 & $(19.0)$ & 2 & $(18.2)$ & 17 & $(19.1)$ & 1.00 \\
\hline K. quasipneumoniae & 8 & $(8.0)$ & 1 & $(9.1)$ & 7 & $(7.9)$ & 1.00 \\
\hline K1 serotype & 14 & $(14.0)$ & 3 & $(27.3)$ & 11 & $(12.4)$ & 0.18 \\
\hline K2 serotype & 13 & $(13.0)$ & 0 & $(0)$ & 13 & $(14.6)$ & 0.35 \\
\hline Hypermucoviscous phenotype & 23 & $(23.0)$ & 3 & $(27.3)$ & 20 & $(22.5)$ & 0.71 \\
\hline
\end{tabular}

CRBSI Catheter-related bloodstream infection

K1 serotype strain has a higher prevalence of $k f u$ than the non-K1 serotype [26, 27]. In this study, K. pneumoniae belonging to the $\mathrm{K} 1$ serotype carried $k f u$ in all strains $(16 / 16,100 \%)$, whereas the K2 serotype had quite low rates $(3 / 13,23.0 \%)$. However, no $K$. variicola isolates were $\mathrm{K} 1$ serotypes. The carriage rates of iutA and $y b t S$, which produce aerobactin and yersiniabactin,

Table 5 Multivariate predictors of 30-day mortality among patients with bloodstream infections

\begin{tabular}{lllll}
\hline & Odds ratio & $95 \% \mathrm{Cl}$ & $p$-value & \\
\hline Liver cirrhosis & 6.43 & $1.0-37.0$ & $<0.05$ & $*$ \\
Mental disorder & 15.00 & $2.33-97.1$ & $<0.005$ & $*$ \\
Skin and soft-tissue infection & 13.16 & $1.19-145.0$ & $<0.05$ & $*$ \\
\hline
\end{tabular}

Cl Confidence interval respectively, were significantly higher in $K$. pneumoniae isolates than in $K$. variicola or $K$. quasipneumoniae isolates [9, 28, 29]. Moreover, entB was found in almost all $K$. pneumoniae and $K$. variicola isolates but only in $36.4 \%$ of $K$. quasipneumoniae isolates. These results suggested that $K$. quasipneumoniae isolates had fewer virulence genes associated with iron acquisition than $K$. pneumoniae or K. variicola isolates, as has been previously reported $[8,29-31]$. On the other hand, the carriage of alls, which is associated with allantoin metabolism, was significantly higher in $K$. quasipneumoniae isolates $(6 / 11,54.5 \%)$ than in $K$. pneumoniae $(21 / 87,24.1 \%)$ or $K$. variicola $(1 / 21,4.8 \%)$ isolates. These results suggested that different mechanisms could contribute in part to the pathogenicity of BSI caused by three these organisms. 
In this study, there were no significant differences between the three organisms in terms of clinical characteristics and impact on outcome, except for the fact that $K$. variicola was more frequently detected in elderly patients, and $K$. quasipneumoniae was found more frequently than $K$. pneumoniae and $K$. variicola in patients with malignancy in Japan. In comparing patients' backgrounds between community-acquired and hospitalacquired infections, ESBL-producing isolates and patients with malignancies were more frequently associated with BSIs in the patients who had hospital-acquired infections. Indeed, these differences have been evaluated in previous reports; however, in most studies $K$. variicola and $K$. quasipneumoniae have unfortunately been misidentified as K. pneumoniae [32-35]. Our study showed that there was no significant difference in the rates of BSIs caused by the three types of Klebsiella species in patients with both community- and hospital-acquired infections.

Moreover, differences in bacterial properties and organisms were not identified as independent risk factors for 30-day mortality. One study showed that patients with $K$. variicola BSI have an increased risk of mortality compared with patients with K. pneumoniae or K. quasipneumoniae BSI in Sweden [19]. This contradiction probably reflects differences in bacterial populations due to the different geographical areas. In the Swedish study, there were fewer isolates belonging to $\mathrm{K} 1(1.4 \%)$ or $\mathrm{K} 2(5.0 \%)$ serotypes than in our study, which had $13.5 \%$ K1 serotype and $10.9 \%$ K2 serotype. $\mathrm{K} 1$ and $\mathrm{K} 2$ serotypes are frequently detected in BSIs, particularly in Asia and South Africa, but less frequently in Europe and North America [36]. Several reports have shown that K1 serotype strains, especially those belonging to clonal complex 23, have a high prevalence of plasmids encoding virulence genes, such as $\operatorname{rmp} A$, inuA, $k f u$ and alls [27, 37, 38]. Furthermore, such K1 $K$. pneumoniae strains are known as hypervirulent $K$. pneumoniae. There have been a few reports with detailed surveillance data on the distribution of capsular serotypes among $K$. pneumoniae isolates derived from patients with invasive infections across Japan [39]. Our study showed hypervirulent $\mathrm{K} 1 \mathrm{~K}$. pneumoniae is frequently the cause of BSI but is not associated with mortality; similar results have been found in Taiwan and China [40, 41]. It is reported that ESBL-producing isolates are associated with higher mortality rates [42]. However, in our study, ESBL-producing isolates were confirmed in only 3 of the 119 isolates. Therefore, depending on the geographical area, additional identification of $K$. variicola isolates from $K$. pneumoniae in routine laboratory tests may not help to lower the mortality rate of patients with BSI caused by Klebsiella species.
In this study, the limitations were the small sample size and low rate of mortality. A large sample size is needed to obtain better evaluation of accuracy, the prevalence of virulence genes, and further information on the differences in the clinical characteristics between $K$. pneumoniae, $K$. variicola, and $K$. quasipneumoniae infections.

\section{Conclusions}

We identified $K$. variicola and $K$. quasipneumoniae using parC sequence-based analysis from isolates initially identified as $K$. pneumoniae obtained from patients with BSI. We found differences in the bacterial characteristics, including the prevalence of virulence genes and phenotypic features between these three phylogroups. BSIs caused by $K$. variicola were significantly more prevalent than those caused by the other two organisms in patients over 80 years old. However, there were no significant differences in 30-day mortality rates. Our findings provided evidence of the differences in bacterial pathogenicity and clinical features among these three phylogroups. Further research may help to determine additional clinical characteristics between these three organisms in patients with BSIs.

\section{Supplementary information}

Supplementary information accompanies this paper at https://doi.org/10. 1186/s12879-019-4498-X.

\begin{abstract}
Additional file 1: Figure S1. Phylogenetic tree based on $314 \mathrm{bp}$ parC gene of 119 Klebsiella clinical isolates and reference strains. The parC gene sequence of reference strains were imported from GenBank; $K$. pneumoniae strain ATCC 35657 (CP015134.1), K. pneumoniae strain ATCC 43816 (CP009208.1), K. pneumoniae strain SC-7 (CP030269.1), K. pneumoniae strain BAA-2146 (CP006659.2), K. pneumoniae strain NUHL 30457 (CP026586.1), K. variicola strain 13,450 (CP030173.1), K. variicola strain GJ3 (CP017289.1), K. quasipneumoniae strain ATCC 700603 (CP029597.1) and E. cloacae strain AR 0072 (CP026850.1). The phylogenetic tree was constructed by the neighbor-joining method and the reliability of the topology of each tree was checked by 500 bootstrap replications.

Additional file 2: Table S1. Summary of all clinical and bacterial characteristics in this study. Table S2. Differences in bacterial characteristics between isolates of hypermucoviscous and non-hypermucoviscous phenotypes. Table S3. Differences in in the prevalence of virulence genes and serotypes of isolates between hospital-acquired and community-acquired infection groups. Table S4. Differences in the prevalence of virulence genes and serotypes of isolates based on clinical characteristics.
\end{abstract}

\section{Abbreviations}

BSIs: Bloodstream infections; CLSI: Clinical and laboratory standards institute; ESBL: Extended spectrum beta-lactamase; MALDI-TOF MS: Matrix-assisted laser desorption ionization-time of flight mass spectrometry; parC: Subunit $C$ of topoisomerase IV

\section{Acknowledgments}

Not applicable.

Authors' contributions

$\mathrm{KI}, \mathrm{NT}, \mathrm{NI}$, and TMa, study conception and design; MK, TK, ST, YT, and YE, collecting isolates and performed the experiments; $\mathrm{KI}, \mathrm{NI}, \mathrm{NT}$, and JS, data gathering, data analysis, manuscript drafting, and editing; IK, TMu, KM, and 
SM, study supervision and manuscript revision; NT and TMa, project administration. All authors read and approved the final manuscript.

\section{Funding}

This research did not receive any specific grant from funding agencies in the public, commercial, or not-for-profit sectors.

\section{Availability of data and materials}

All data generated or analyzed during this study are included in this published article and supplementary files.

\section{Ethics approval and consent to participate}

Ethical clearances were obtained from Institutional Ethical Review Board of Saitama Medical University Hospital and Saitama Medical University International Medical Center (approval numbers 17-127 and 17-275). Both institutional ethical review boards approved the opt-out consent process. All individuals were given the opportunity to decline participation in this study through an opt-out consent process. Kazuo Imai and Noriomi Ishibashi were granted permission to access the raw clinical data by Institutional Ethical Review Board of Saitama Medical University Hospital and Saitama Medical University International Medical Center, respectively. Confidentiality was maintained by avoiding the use of names or other identifiers.

\section{Consent for publication}

Not applicable.

\section{Competing interests}

The authors declare that they have no conflicts of interests.

\section{Author details}

${ }^{1}$ Department of Infectious Disease and Infection Control, Saitama Medical University, 38 Morohongo, Moroyama-machi, Iruma-gun, Saitama 350-0495, Japan. ${ }^{2}$ Center for Clinical Infectious Diseases and Research, Saitama Medical University, 38 Morohongo, Moroyama-machi, Iruma-gun, Saitama 350-0495, Japan. ${ }^{3}$ Infectious Diseases and Infection Control, Saitama Medical University International Medical Center, 1-1397 Yamane, Hidaka, Saitama 350-1298, Japan. ${ }^{4}$ Clinical Laboratory Medicine, Saitama Medical University Hospital, 38 Morohongo, Moroyama-machi, Iruma-gun, Saitama 350-0495, Japan. ${ }^{5}$ Department of Clinical Laboratory Medicine, Saitama Medical University International Medical Center, 1-1397 Yamane, Hidaka, Saitama 350-1298, Japan. ${ }^{6}$ Department of Microbiology, Saitama Medical University, 38 Morohongo, Moroyama-machi, Iruma-gun, Saitama 350-0495, Japan.

\section{Received: 22 July 2018 Accepted: 23 September 2019}

\section{Published online: 08 November 2019}

\section{References}

1. Donnenberg MS. Enterobacteriaceae. In: Mandell GL, Bennett JE, Dolin R, editors. Mandell, Douglas, and Bennett's Principles and practice of infectious diseases. 7th ed. Philadelphia; 2010.

2. Lee HC, Chuang YC, Yu WL, Lee NY, Chang CM, Ko NY, Wang LR, Ko WC. Clinical implications of hypermucoviscosity phenotype in Klebsiella pneumoniae isolates: association with invasive syndrome in patients with community-acquired bacteraemia. J Intern Med. 2006;259:606-14.

3. Shon AS, Bajwa RP, Russo TA. Hypervirulent (hypermucoviscous) Klebsiella pneumoniae: a new and dangerous breed. Virulence. 2013;4:107-18.

4. Siu LK, Yeh KM, Lin JC, Fung CP, Chang FY. Klebsiella pneumoniae liver abscess: a new invasive syndrome. Lancet Infect Dis. 2012;12:881-7.

5. Yeh KM, Kurup A, Siu LK, Koh YL, Fung CP, Lin JC, Chen TL, Chang FY, Koh $\mathrm{TH}$. Capsular serotype K1 or K2, rather than magA and rmpA, is a major virulence determinant for Klebsiella pneumoniae liver abscess in Singapore and Taiwan. J Clin Microbiol. 2007:45:466-71.

6. Fang CT, Chuang YP, Shun CT, Chang SC, Wang JT. A novel virulence gene in Klebsiella pneumoniae strains causing primary liver abscess and septic metastatic complications. J Exp Med. 2004;199:697-705.

7. Yu WL, Ko WC, Cheng KC, Lee HC, Ke DS, Lee CC, Fung CP, Chuang YC. Association between rmpA and magA genes and clinical syndromes caused by Klebsiella pneumoniae in Taiwan. Clin Infect Dis. 2006;42:1351-8.

8. Podschun R, Fischer A, Ullmann U. Siderophore production of Klebsiella species isolated from different sources. Zentralbl Bakteriol. 1992;276:481-6.
9. Podschun R, Sievers D, Fischer A, Ullmann U. Serotypes, hemagglutinins, siderophore synthesis, and serum resistance of Klebsiella isolates causing human urinary tract infections. J Infect Dis. 1993;168:1415-21.

10. Ma LC, Fang $C T$, Lee $C Z$, Shun $C T$, Wang JT. Genomic heterogeneity in Klebsiella pneumoniae strains is associated with primary pyogenic liver abscess and metastatic infection. J Infect Dis. 2005;192:117-28.

11. Jagnow J, Clegg S. Klebsiella pneumoniae MrkD-mediated biofilm formation on extracellular matrix- and collagen-coated surfaces. Microbiology. 2003; 149:2397-405.

12. Chou HC, Lee CZ, Ma LC, Fang CT, Chang SC, Wang JT. Isolation of a chromosomal region of Klebsiella pneumoniae associated with allantoin metabolism and liver infection. Infect Immun. 2004;72:3783-92.

13. Rosenblueth M, Martinez L, Silva J, Martinez-Romero E. Klebsiella variicola, a novel species with clinical and plant-associated isolates. Syst Appl Microbiol. 2004;27:27-35.

14. Brisse S, Passet V, Grimont PA. Description of Klebsiella quasipneumoniae sp. nov., isolated from human infections, with two subspecies, Klebsiella quasipneumoniae subsp. quasipneumoniae subsp. nov. and Klebsiella quasipneumoniae subsp. similipneumoniae subsp. nov., and demonstration that Klebsiella singaporensis is a junior heterotypic synonym of Klebsiella variicola. Int J Syst Evol Microbiol. 2014;64:3146-52.

15. Berry GJ, Loeffelholz MJ, Williams-Bouyer N. An investigation into laboratory misidentification of a bloodstream Klebsiella variicola infection. J Clin Microbiol. 2015;53:2793-4.

16. Long SW, Linson SE, Ojeda Saavedra M, Cantu C, Davis JJ, Brettin T, Olsen RJ. Whole-genome sequencing of human clinical Klebsiella pneumoniae isolates reveals misidentification and misunderstandings of Klebsiella pneumoniae, Klebsiella variicola, and Klebsiella quasipneumoniae. mSphere. 2017;2:e00290.

17. Seki M, Gotoh K, Nakamura S, Akeda Y, Yoshii T, Miyaguchi S, Inohara H, Horii T, Oishi K, lida T, et al. Fatal sepsis caused by an unusual Klebsiella species that was misidentified by an automated identification system. J Med Microbiol. 2013;62:801-3.

18. Fenselau C, Demirev PA. Characterization of intact microorganisms by MALDI mass spectrometry. Mass Spectrom Rev. 2001;20:157-71.

19. Maatallah M, Vading M, Kabir MH, Bakhrouf A, Kalin M, Naucler P, Brisse S, Giske CG. Klebsiella variicola is a frequent cause of bloodstream infection in the stockholm area, and associated with higher mortality compared to $K$. pneumoniae. PLoS One. 2014;9:e113539.

20. Brisse S, Verhoef J. Phylogenetic diversity of Klebsiella pneumoniae and Klebsiella oxytoca clinical isolates revealed by randomly amplified polymorphic DNA, gyrA and parC genes sequencing and automated ribotyping. Int J Syst Evol Microbiol. 2001;51:915-24.

21. Compain F, Babosan A, Brisse S, Genel N, Audo J, Ailloud F, Kassis-Chikhan $\mathrm{N}$, Arlet G, Decre D. Multiplex PCR for detection of seven virulence factors and K1/K2 capsular serotypes of Klebsiella pneumoniae. J Clin Microbiol. 2014:52:4377-80.

22. Holt KE, Wertheim H, Zadoks RN, Baker S, Whitehouse CA, Dance D, Jenney A, Connor TR, Hsu LY, Severin J, et al. Genomic analysis of diversity, population structure, virulence, and antimicrobial resistance in Klebsiella pneumoniae, an urgent threat to public health. Proc Natl Acad Sci U S A. 2015;112:E3574-81.

23. Garza-Ramos U, Silva-Sanchez J, Barrios H, Rodriguez-Medina N, MartinezBarnetche J, Andrade V. Draft genome sequence of the first hypermucoviscous Klebsiella variicola clinical isolate. Genome Announc. 2015;3:e01352.

24. Garza-Ramos U, Silva-Sanchez J, Catalan-Najera J, Barrios H, RodriguezMedina N, Garza-Gonzalez E, Cevallos MA, Lozano L. Draft Genome Sequence of a Hypermucoviscous extended-spectrum-beta-lactamaseproducing Klebsiella quasipneumoniae subsp. similipneumoniae clinical isolate. Genome Announc. 2016;4:e00475.

25. Arena F, Henrici De Angelis L, Pieralli F, Di Pilato V, Giani T, Torricelli F, D'Andrea MM, Rossolini GM. Draft genome sequence of the first hypermucoviscous Klebsiella quasipneumoniae subsp. quasipneumoniae isolate from a bloodstream infection. Genome Announc. 2015;3:e00952.

26. Fang CT, Lai SY, Yi WC, Hsueh PR, Liu KL, Chang SC. Klebsiella pneumoniae genotype K1: an emerging pathogen that causes septic ocular or central nervous system complications from pyogenic liver abscess. Clin Infect Dis. 2007:45:284-93.

27. Struve C, Roe CC, Stegger M, Stahlhut SG, Hansen DS, Engelthaler DM, Andersen PS, Driebe EM, Keim P, Krogfelt KA. Mapping the evolution of hypervirulent Klebsiella pneumoniae. MBio. 2015;6:e00630. 
28. Nassif X, Sansonetti PJ. Correlation of the virulence of Klebsiella pneumoniae $\mathrm{K} 1$ and $\mathrm{K} 2$ with the presence of a plasmid encoding aerobactin. Infect Immun. 1986;54:603-8.

29. Tarkkanen AM, Allen BL, Williams PH, Kauppi M, Haahtela K, Siitonen A Orskov I, Orskov F, Clegg S, Korhonen TK. Fimbriation, capsulation, and ironscavenging systems of Klebsiella strains associated with human urinary tract infection. Infect Immun. 1992;60:1187-92.

30. Miles AA, Khimji PL. Enterobacterial chelators of iron: their occurrence, detection, and relation to pathogenicity. J Med Microbiol. 1975;8:477-90.

31. Lodge JM, Williams P, Brown MR. Influence of growth rate and iron limitation on the expression of outer membrane proteins and enterobactin by Klebsiella pneumoniae grown in continuous culture. J Bacteriol. 1986;165:353-6.

32. Jung Y, Lee MJ, Sin HY, Kim NH, Hwang JH, Park J, Choe PG, Park WB, Kim ES, Park SW, et al. Differences in characteristics between healthcareassociated and community-acquired infection in community-onset Klebsiella pneumoniae bloodstream infection in Korea. BMC Infect Dis. 2012;12:239.

33. Wu HS, Wang FD, Tseng CP, Wu TH, Lin YT, Fung CP. Characteristics of healthcare-associated and community-acquired Klebsiella pneumoniae bacteremia in Taiwan. J Inf Secur. 2012;64:162-8.

34. Tsay RW, Siu LK, Fung CP, Chang FY. Characteristics of bacteremia between community-acquired and nosocomial Klebsiella pneumoniae infection: risk factor for mortality and the impact of capsular serotypes as a herald for community-acquired infection. Arch Intern Med. 2002;162:1021-7.

35. Paterson DL, Ko WC, Von Gottberg A, Mohapatra S, Casellas JM, Goossens H, Mulazimoglu L, Trenholme G, Klugman KP, Bonomo RA, et al. International prospective study of Klebsiella pneumoniae bacteremia: implications of extended-spectrum beta-lactamase production in nosocomial infections. Ann Intern Med. 2004;140:26-32.

36. Yu VL, Hansen DS, Ko WC, Sagnimeni A, Klugman KP, von Gottberg A, Goossens H, Wagener MM, Benedi VJ. Virulence characteristics of Klebsiella and clinical manifestations of K. pneumoniae bloodstream infections. Emerg Infect Dis. 2007;13:986-93.

37. Luo Y, Wang Y, Ye L, Yang J. Molecular epidemiology and virulence factors of pyogenic liver abscess causing Klebsiella pneumoniae in China. Clin Microbiol Infect. 2014:20:0818-24.

38. Brisse S, Fevre C, Passet V, Issenhuth-Jeanjean S, Tournebize R, Diancourt L, Grimont P. Virulent clones of Klebsiella pneumoniae: identification and evolutionary scenario based on genomic and phenotypic characterization. PLoS One. 2009:4:e4982.

39. Ito R, Shindo Y, Kobayashi D, Ando M, Jin W, Wachino J, Yamada K, Kimura K, Yagi T, Hasegawa Y, et al. Molecular epidemiological characteristics of Klebsiella pneumoniae associated with bacteremia among patients with pneumonia. J Clin Microbiol. 2015;53:879-86.

40. Liao CH, Huang YT, Lai CC, Chang CY, Chu FY, Hsu MS, Hsu HS, Hseuh PR. Klebsiella pneumoniae bacteremia and capsular serotypes, Taiwan. Emerg Infect Dis. 2011;17:1113-5.

41. Liu YM, Li BB, Zhang YY, Zhang W, Shen H, Li H, Cao B. Clinical and molecular characteristics of emerging hypervirulent Klebsiella pneumoniae bloodstream infections in mainland China. Antimicrob Agents Chemother. 2014:58:5379-85.

42. Kang Cl, Kim SH, Park WB, Lee KD, Kim HB, Kim EC, Oh MD, Choe KW. Bloodstream infections due to extended-spectrum beta-lactamaseproducing Escherichia coli and Klebsiella pneumoniae: risk factors for mortality and treatment outcome, with special emphasis on antimicrobial therapy. Antimicrob Agents Chemother. 2004;48:4574-81.

\section{Publisher's Note}

Springer Nature remains neutral with regard to jurisdictional claims in published maps and institutional affiliations.

Ready to submit your research? Choose BMC and benefit from:

- fast, convenient online submission

- thorough peer review by experienced researchers in your field

- rapid publication on acceptance

- support for research data, including large and complex data types

- gold Open Access which fosters wider collaboration and increased citations

- maximum visibility for your research: over $100 \mathrm{M}$ website views per year

At $\mathrm{BMC}$, research is always in progress.

Learn more biomedcentral.com/submissions 